\title{
Reinforcing the Team: A Call to Critical Care Nephrology in the COVID-19 Epidemic
}

\author{
Lilia Maria Rizo-Topete $^{a, b} \quad$ Faeq Husain-Syed ${ }^{c} \quad$ Claudio Ronco $^{d, e, f}$ \\ aDepartment of Internal Medicine, Nephrology Service, Hospital Universitario "José Eleuterio González", Monterrey, \\ Mexico; ${ }^{b}$ Department of Internal Medicine, Hospital Christus Muguerza Alta Especialidad, Monterrey, Mexico; \\ 'Department of Internal Medicine II, Division of Nephrology, Pulmonology and Critical Care Medicine, Giessen \\ and Marburg Lung Centre (UGMLC), Member of the German Centre for Lung Research (DZL), University Hospital \\ Giessen and Marburg, Giessen, Germany; 'Department of Medicine (DIMED), Università di Padova, Padova, Italy;

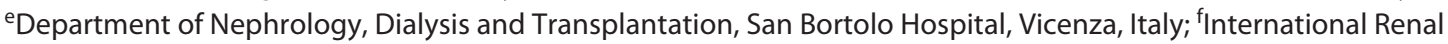 \\ Research Institute of Vicenza (IRRIV), Vicenza, Italy
}

Keywords

COVID-19 · Critical care nephrology · Acute kidney injury

\section{Background}

In the current global climate, humanity is at one of its most vulnerable moments. The coronavirus epidemic is one of the biggest disasters to hit healthcare systems worldwide, and health professionals are in a situation where they have to derive the best outcomes from the available resources.

This pandemic is not the first one to cripple humanity. Not so long ago in Mexico, in 2009, more than 2,000 patients were affected by an influenza pandemic [1]. This influenza A (H1N1), was catastrophic and required the healthcare sector to resort to desperate interventions. This episode demonstrated the importance of being organized; for example, it was in this situation that the extracorporeal membrane oxygenator reemerged as a means of treatment in patients with severe respiratory disease because mechanical ventilation was insufficient to treat refractory hypercapnia and hypoxia $[2,3]$.
Now, 10 years later, we are witness to the coronavirus disease 2019 (COVID-19) pandemic that originated in Wuhan, China. Its high morbidity and relatively high mortality rates were a shock, and a reminder that critical care teams need to work together and collaborate with experts from around the globe in order to achieve the desirable results, like the use of hemoperfusions and extracorporeal therapies proposed as alternative treatments to attack the cytokine storms associated with the disease [4]. This commentary attempts to the necessity to incorporate well-structured critical care teams from the beginning for treating patients with SARS-CoV-2, mainly in the multiorgan support therapy scenario.

\section{Critical Care Nephrology}

Acute kidney injury (AKI) is common among acutely ill patients. In COVID 19, the incidence is not clear and may be underestimated. But even patients who do not have AKI, occasionally, require extracorporeal therapies, such as hepatic support, plasma exchange, and more recently, cytokine removal through adsorbents [4-7]. Ne- 


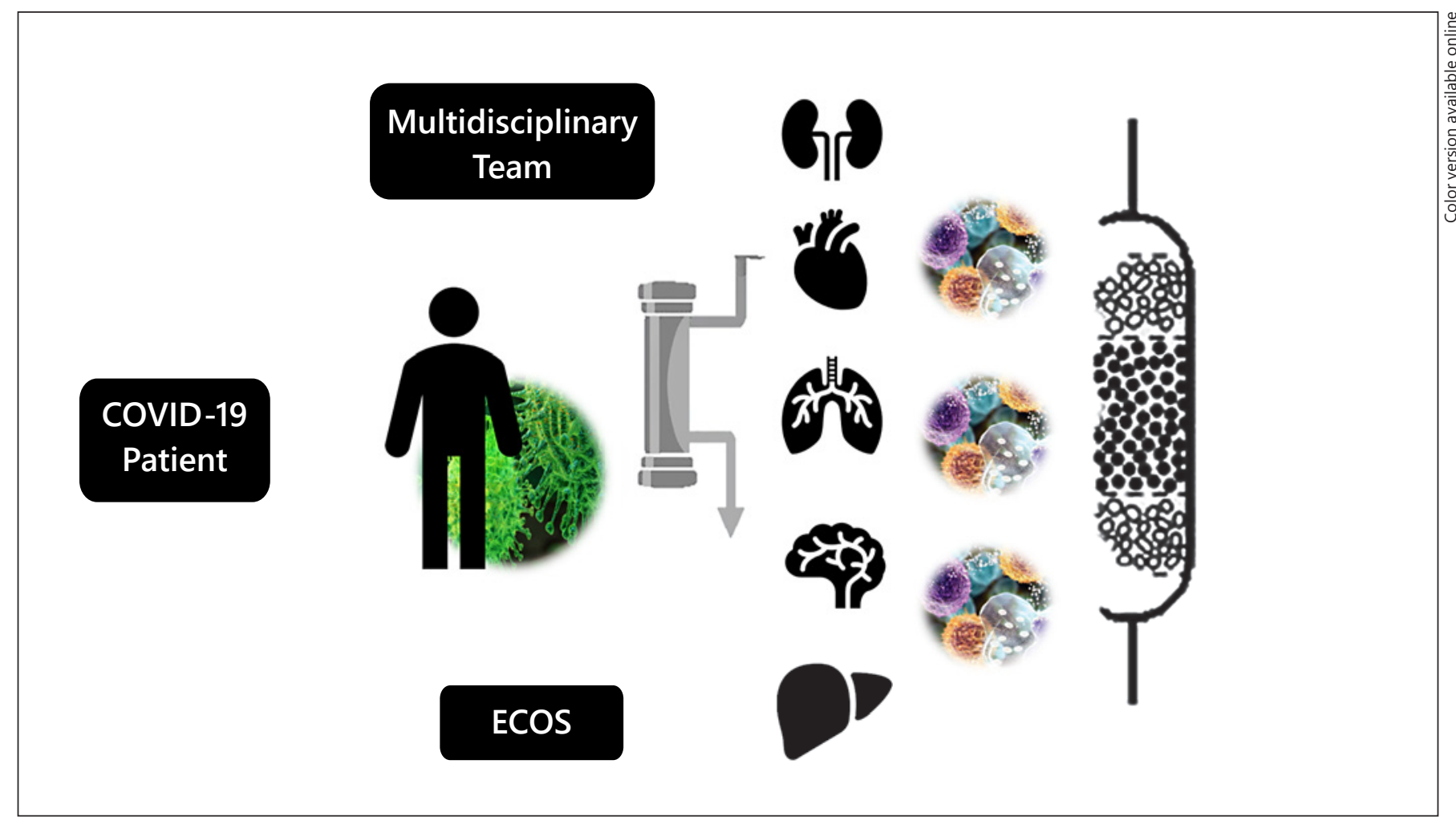

Fig. 1. Artificial organ cross talk could need the attention of more than one expert. The good communication and good clinical practice with patient at the centre of the take decision table should be encouraged. In COVID-19 patients, new therapies are being pro- posed and discussed, like ECOS and sorbents, because of the necessity of a strong and well-coordinated teamwork. COVID-19, coronavirus disease 2019; ECOS, extracorporeal organ support. phrologists have consistently focused on the association between AKI stages and patient outcomes $[8,9]$, and it was recently reported that $\mathrm{AKI}$ is linked to a wide range of distant organ injuries and high mortality rates [10]. In the context of pneumonia, it has been reported that $30 \%$ of patients with severe pneumonia develop AKI, and most of them require renal replacement therapy (RRT) [3]. Critical care nephrology (CCN) is a new field in medicine that focuses on the issues related to the management and prevention of AKI and the associated consequences [11]. The aim of CCN is to provide integrated multidisciplinary care with coordinated efforts so that patients can be provided with safe care by physicians with enough expertise $[11,12]$.

The field of general critical care has evolved into a subspecialty that provides care to patients with a high number of comorbidities; they require prolonged care and have a high incidence of multiorgan failure that requires extracorporeal multiorgan support systems. In the last 2 decades, there has been significant progress in research on the interactions between native organs and extracorporeal multiorgan support systems [13]. Given the increase in the variety and complexity of artificial organ support systems in the intensive care unit (ICU), it is very important to adhere to the first principle of medicine, that is, primum non nocere or "first, do no harm." To this end, there is a critical need to build healthcare teams with significant expertise in order to provide safe and efficient care to critically ill patients. Such teams are particularly necessary for patients who require extracorporeal organ support (ECOS) in the context of multiple organ dysfunction, as has been described in severe COVID-19 cases [4] (shown in Fig. 1). These teams provide platforms for healthcare workers to share their knowledge and experience for solving ethical dilemmas regarding end-of-life care or palliative care so that better medical care can be provided and the quality of life of the patients and their families can be improved. In particular, in the framework of precision medicine, timely and accurate communication between team members is important. 
Previously, over $50 \%$ of patients developed stage 1 AKI at some point during their ICU stay, whereas the requirement for RRT is only 5-10\% [14]. Despite the lower rate of RRT in comparison with the incidence of AKI, RRT is the most commonly used form of ECOS in the ICU. Additionally, $50-65 \%$ of patients treated with the extracorporeal membrane oxygenator develop AKI that requires RRT [15]. Therefore, nephrologists, as experts with a deep knowledge of physiology and extracorporeal blood purification techniques, are vital members of critical care teams. Commonly, nephrologists are called to manage AKI in cases of oliguria, uremia, and fluid overload associated with RRT. Maybe nephrologists are consulted too late in the case of AKI patients. We propose the development of a nephrology rapid response team for the care of high-risk patients, and COVID-19 patients are per se high-risk patients (diabetes, hypertension, obesity, advanced age, or other chronic illness). This team would incorporate protocols in the form of flowcharts for the early recognition (e.g., by the use of AKI biomarkers such as tissue inhibitor of metalloproteinase- 2 and insulinelike growth factor-binding protein 7) and provision of better-quality preventive measures, including ECOS [1618]. Their inclusion would also help improve interdepartmental cooperation. At these days, the incidence of AKI in COVID-19 patients goes around $20-40 \%$ in ICU patients as statistics from Europe and USA report [19], once again we are arriving late.

Nephrologists are also experienced in the use of hemoperfusion and adsorbtion techniques in combination with continuous renal replacement therapy (CRRT) for the removal of inflammatory cells. As it is known that the coronavirus generates an inflammatory response in the lungs called the "cytokine storm syndrome" (4), nephrologists may be able to step in and provide guidance on appropriate management and obtaining better outcomes in critical cases. Thus, the integration of nephrologists in the treatment of complicated cases of COVID-19-associated pneumonia might help to save more lives.

CRRT can be used in conjunction with adsorbent membranes, such as oXiris, or adsorbent cartridges, such as CytoSorb, for the removal of cytokines. This combination might prove effective and could be used as the frontline treatment when possible. However, in some emerging economies, opportunities for the use of such a combined technique could be limited. Nonetheless, CCN can always help incorporating knowledge and innovations, such as the use of Theranova filters, to help patients who are on conventional hemodialysis machines or CRRT.

Critical Care Nephrology in COVID-19

\section{Conclusion}

We propose the early inclusion of $\mathrm{CCN}$ in critical care teams as an essential multidisciplinary working group for the management of patients who require CRRT and ECOS. The critical care nephrologists could mediate between multiple organ support therapies, particularly in cases where RRT is being considered or has been started. The COVID-19 pandemic is a special situation that requires healthcare teams to work side by side, as a united army of soldiers, and utilize each other's expertise to develop new strategies for the treatment of this infection.

\section{Conflict of Interest Statement}

In the las 3 years, Claudio Ronco has been consulting or part of advisory board ASAHI, Astute, Baxter, Biomerieux, B. Braun, CytoSorbents, ESTOR, FMC, GE, Jafron, Medtronic, and Toray. The other authors have no conflicts of interest to declare.

\section{Funding Sources}

The authors declare that they have no funding to declare.

\section{Author Contributions}

L.RT., F.H.-S., and C.R. were involved in drafting, writing, and editing the manuscript. L.RT. conceived the concept underlying the manuscript and is the senior author of the manuscript. All authors have read and approved the final version of the manuscript.

References

1 Chowell G, Bertozzi SM, Colchero MA, Lopez-Gatell H, Alpuche-Aranda C, Hernandez $M$, et al. Severe respiratory disease concurrent with the circulation of H1N1 influenza. N Engl J Med. 2009 Aug 13;361(7):674-9.

2 Clinical aspects of pandemic 2009 influenza A (H1N1) virus infection. N Engl J Med. 2010 May 6;362(18):1708-19.

3 Perez-Padilla R, de la Rosa-Zamboni D, Ponce de Leon S, Hernandez M, QuiñonesFalconi F, Bautista E, et al. Pneumonia and respiratory failure from swine-origin influenza A (H1N1) in Mexico. N Engl J Med. 2009 Aug 13;361(7):680-9.

4 Ronco C, Reis T, De Rosa S. Coronavirus epidemic and extracorporeal therapies in intensive care: $s i$ vis pacem para bellum. Blood $\mathrm{Pu}$ rif. 2020;49(3):255-258.

5 Bellomo R, Ronco C, Mehta RL, Asfar P, Helms JB, Darmon M, et al. Acute kidney injury in the ICU : from injury to recovery: reports from the 5th Paris international conference. Ann Intensive Care. 2017;7(1):49. 
6 IFF, Puci MV, Ferraro OE, Husain-syed F, Rizo-topete L, Senzolo M, et al. Development and validation of quick Acute Kidney Injuryscore (q-AKI) to predict acute kidney injury at admission to a multidisciplinary intensive care unit. PLoS One. 2019;14(6):e0217424.

7 Thomas ME, Blaine C, Dawnay A, Devonald MA, Ftouh S, Laing C, et al. The definition of acute kidney injury and its use in practice. Kidney Int. 2015;87(1):62-73.

8 Uchino S, Bellomo R, Goldsmith D, Bates S, Ronco C. An assessment of the RIFLE criteria for acute renal failure in hospitalized patients. Crit Care Med. 2006;34(7):1913.

9 Hoste EA, Bagshaw SM, Bellomo R, Cely CM, Colman R, Cruz DN, et al. Epidemiology of acute kidney injury in critically ill patients: the multinational AKI-EPI study. Intensive Care Med. 2015;41(8):1411-23.

10 Mehta RL, Soroko SB, Ikizler TA. Sepsis as a cause and consequence of acute kidney injury: program to improve care in acute renal disease; 2011. Vols. 241-8.
11 Ronco C, Bellomo R, Feriani M, La Greca G. Critical care nephrology: the time has come. Kidney Int Suppl. 1998 Feb 1;66(2):S1-2.

12 Rizo-Topete L, Ronco C. Critical care nephrology: a multidisciplinary approach. Blood Purif. 2017;43(1-3):53.

13 Husain-syed F, Ricci Z, Brodie D, Vincent J, Ranieri VM, Slutsky AS, et al. Extracorporeal organ support (ECOS) in critical illness and acute kidney injury: from native to artificial organ crosstalk; 2018.

14 Kellum JA, Murugan R. Effects of non-severe acute kidney injury on clinical outcomes in critically ill patients. Crit Care. 2016;20(1): 159.

15 Kielstein JT, Heiden AM, Beutel G, Gottlieb J, Wiesner $\mathrm{O}$, Hafer $\mathrm{C}$, et al. Renal function and survival in 200 patients undergoing ECMO therapy; 2013 Nov. p. 86-90.
16 Digvijay K, Ferrari F, Brendolan A, Raffaele B, Giavarina D. Tissue inhibitor metalloproteinase-2 (TIMP-2). IGF-binding protein-7 (IGFBP7) levels are associated with adverse outcomes in patients in the intensive care unit with acute kidney injury. Kidney Int. 2019; 95(6): 1486-93.

17 Rizo-Topete LM, Rosner MH, Ronco C. Acute kidney injury risk assessment and the nephrology rapid response team. Blood Purif. 2017;43(1-3):82.

18 Ronco C, Rizo-Topete L, Serrano-Soto M, Kashani K. Pro: prevention of acute kidney injury: time for teamwork and new biomarkers. Nephrol Dial Transplant. 2017;32(3): 408

19 Ronco C, Reis T, Husain-Syed F. Management of acute kidney injury in patients with COVID-19. Lancet Respir Med. 2020 Jul;8(7): $738-42$. 